http://jmscr.igmpublication.org/home/

ISSN (e)-2347-176x ISSN (p) 2455-0450

crossref DOI: https://dx.doi.org/10.18535/jmscr/v9i5.33

Journal Of Medical Science And Clinical Research

IGM Publication

An official Publication of IGM Publication

\title{
Blast Crisis Presenting as Ischemic Stroke in a Young Female
}

Authors

Jwala K A ${ }^{1}$, Sukanya M K ${ }^{2}$, Shahir M Kabir ${ }^{3}$, Suresh Raghavan ${ }^{4}$, Lijo K Mathew

${ }^{1}$ Junior Resident, ${ }^{2}$ Junior Resident, ${ }^{3}$ Junior Resident, ${ }^{4}$ Professor, ${ }^{5}$ Associate Professor

Department of general Medicine, Govt. Medical College, Kottayam, Kerala, India

*Correspondence Author

Jwala K A

Junior Resident, Department of General Medicine, Govt.Medical College, Kottayam, Kerala, India

\section{Abstract}

Acute Myeloid leukemia usually presents with complaints like fatigue, lethargy, weight loss, dyspnea, bleeding manifestations, bone pain, infections etc. Neurological manifestations as initial presentation is rare in acute leukemia. Among neurological manifestations, Ischemic strokes are even more rare in acute leukemia. Herein we report a rare case of an eighteen year old female with no previous illness who presented with left middle cerebral artery infarct as the initial presentation of acute Myeloidleukemia. Our case points to the importance of investigating for hematological malignancy in a patient presenting as young stroke in the absence of other causes.

\section{Introduction}

Neurological complications can occur in leukemia due to it's primarily hematogenous nature. Many of these complications occur once treatment is started for leukemia. Direct infiltration of brain parenchyma by leukemic cells, meningeal metastasis, hemorrhage, thrombosis due to leukocytosis and resultant hyperviscocity resulting in vascular occlusion, infections due to incompetent immune cells etc are the mechanisms for neurological complications of leukemia.

\section{Case Presentation}

An 18 year old female was admitted with headache of 10 days, progressively worsening and disturbing her sleep and associated with vomiting. She had no fever.
Past history and family history - nothing contributory

She was not on any drugs.

On examination she had severe pallor, was stuporous and opening eyes to painful stimulus. Pupils were equal and reactive to light. Occulocephalic reflex present. She had right sided hemiplegia and right extensor plantar reflex. Abdomen and other systems were within normal limits. Clinically we suspected the possibility of massive young stroke, most probably stem occlusion of left middle cerebral artery. We also considered acute meningitis as it is one of the common cause of young stroke in Indian population. Patient was given anti edima measures, neuro protective agents and other supportive measures, but patient developed seizures and was intubated. 


\section{Investigations}

HB 3.8

MCV 96.9

HCT 12.2

Total count 8520

N 73 L24 M 2

Platelet 15000

Random blood sugar 134

Urea 22

Creatinine 0.9

Sodium, potassium, calcium, magnesium, phosphate - normal

Total bilirubin 1

Direct Bilirubin 0.2

SGOT 28

SGPT 25

Alkaline phosphatise 44

Prothrombin time -15.3

INR 1.15
Activated partial thromboplastin time 25.3 HIV, VDRL, CRP, HBsag, HCB - negative

ANA-IF - negative

Anti double stranded DNA anti body - negative

Lupus anti coagulant, IgM, IgG, antiphospholipid antibody, anticardiolipin antibody - negative

D-dimer - normal

Fibrin degradation products - normal

Bedside echo - no evidence of thrombus or pulmanory embolism.

Bedside USG abdomen - no hepatospleenomegaly Chest X-ray, ECG within normal limits.

MRI brain showed T2 hyper intense signals with difusion restriction involving left insular, left perisylvian fronto palatal lobes and left external capsule, suggestive of left middle cerebal artery territory infarct. no evidence of dural venous sinus thrombosis.

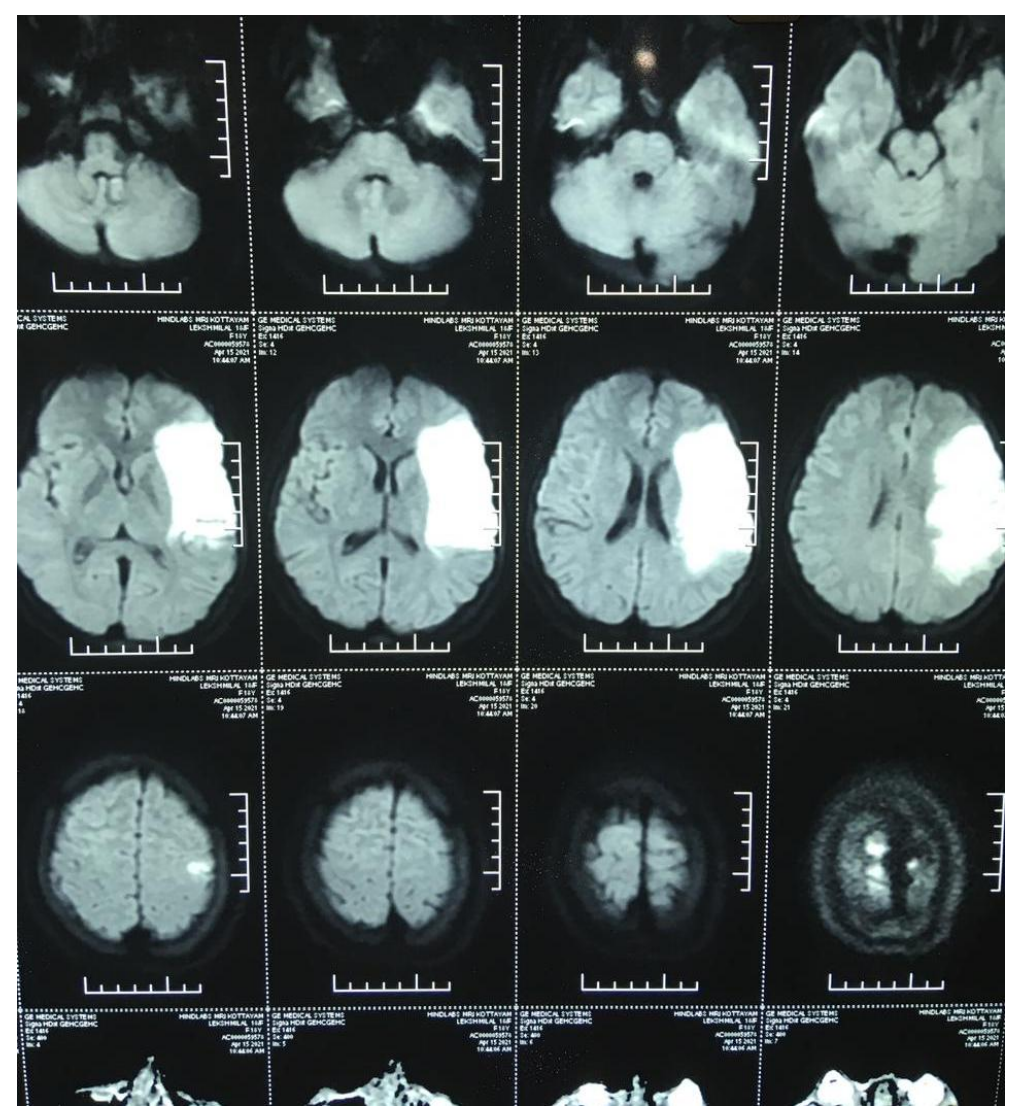

Fig. 1 Peripheral smear reported total WBC count within normal limits 


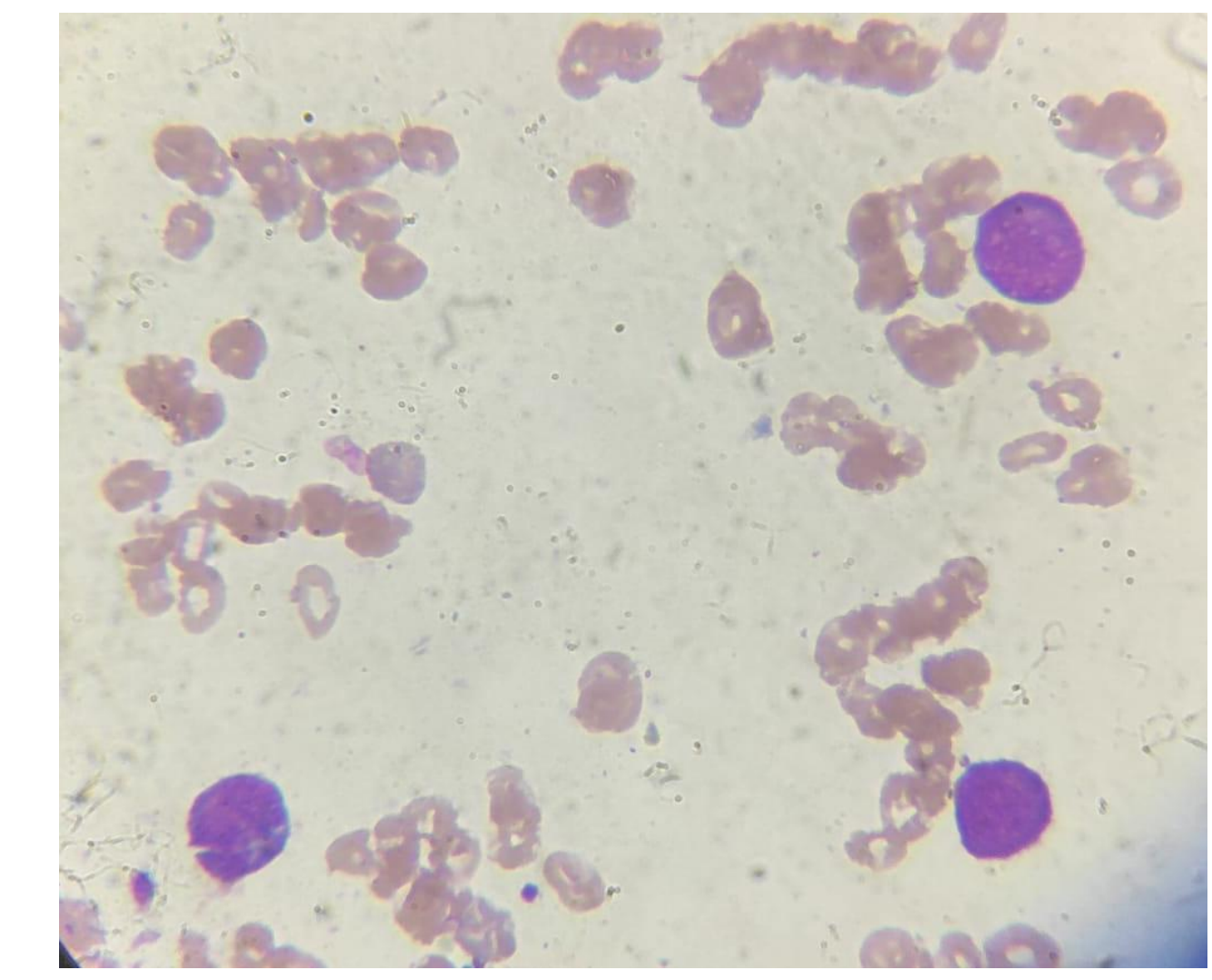

Fig. 2 Differential count

\section{N 4\% L 30\% M 3\% Blast cells 63\% MPO positive}

Peripheral smear report is suggestive of acute Myeloid leukemia

\section{Discussion}

Our patient who presented as left MCA territory stroke on further investigation was found to have acute Myeloid leukemia in blast crisis. She was completely asymptomatic before ten days. Leukemia patients presenting as stroke at initial presentation is rare. Most of the leukemia patients presenting as stroke are having hemorrhagic stroke often due to associated thrombocytopenia. There was no evidence of any infections or drug use for her. Mechanisms of vascular involvement in leukemia are hyperviscocity, leukostasis, disseminated intravascular coagulation, vasculitis, cytokine mediated inflammation. In our patient peripheral total leukocyte counts were normal, hence infarct is unlikely to be due to leukostasis. Infarct is most likely to be due to cytokine mediated vasculitis. Invasive investigation were not done due to the critical state of the patient.
Our case has many rarities. In her, the initial presentation of acute Myeloid leukemia was as massive cerebral infarct. Previous reported cases of acute Myeloid leukemia with cerebral infarct was associated with leukocytosis. But our patient had normal WBC counts. Hematological malignancy like acute leukemia should be considered as a possibility while investigating a case of young stroke even if peripheral blood leukocyte counts are within normal. 